\title{
Norm-conserving pseudopotentials and
}

\section{basis sets optimized for lanthanide}

\section{molecules and solid-state compounds}

\author{
Jun-Bo Lu ${ }^{a, c}$, David C. Cantu ${ }^{b}$, Manh-Thuong Nguyen ${ }^{c}$, Jun Li $^{a^{*}}$, Vassiliki-Alexandra Glezakou ${ }^{c^{*}}$, \\ Roger Rousseau ${ }^{c^{*}}$
}

${ }^{a}$ Department of Chemistry and Key Laboratory of Organic Optoelectronics \& Molecular Engineering of the Ministry of Education, Tsinghua University, Beijing 100084, China; ${ }^{b}$ Chemical and Materials Engineering, University of Nevada, Reno, Reno, Nevada 89557, USA; 'Basic and Applied Molecular Foundations, Pacific Northwest National Laboratory, P.O. Box 999, Richland, Washington 99352, USA

*Corresponding authors: junli@tsinghua.edu.cn, vanda.glezakou@pnnl.gov, roger.rousseau@pnnl.gov

\begin{abstract}
A complete set of pseudopotentials and accompanying basis sets for all lanthanide elements are presented based on the relativistic, norm-conserving, separable, dual-space Gaussian-type pseudopotential protocol of Goedecker, Teter and Hutter (GTH) within the generalized gradient approximation (GGA) and the exchange-correlation functional of Perdew, Burke and Ernzerhof (PBE). The corresponding basis sets have been molecularly optimized (MOLOPT) using a contracted form with a single set of Gaussian exponents for $s, p$ and $d$ states. The $f$ states are uncontracted explicitly with Gaussian exponents. Moreover, the Hubbard U values for each lanthanide element, to be used in $\mathrm{DFT}+\mathrm{U}$ calculations, are also tabulated, allowing for the proper treatment of the strong on-site Coulomb interactions of localized $4 f$ electrons. The accuracy and reliability of our GTH pseudopotentials and companion basis sets optimized for lanthanides is illustrated by a series of test calculations on lanthanide-centered molecules, and solid-state systems, with the most common oxidation states. We anticipate that these pseudopotentials and basis sets will enable larger-scale density functional theory calculations and ab initio molecular dynamics simulations of lanthanide molecules in either gas or condensed phases, as well as of solid state lanthanide-containing materials, allowing to further explore the chemical and physical properties of lanthanide systems.
\end{abstract}




\section{INTRODUCTION}

Research on lanthanide chemistry, physics, and materials is an active area due to the unique properties lanthanides that primarily arise from their extremely localized $4 f$ electrons. ${ }^{1-3}$ The presence of lanthanides in materials results in interesting optical, luminescent, magnetic, or superconducting properties, as well as medical contrast agents, and catalysts. ${ }^{4-14}$ The rapid development of computational technology and electronic structure theory has enabled the modeling of the physical and chemical properties of lanthanide-based systems. Density functional theory (DFT) in particular, when supplemented with the appropriate pseudopotentials, can be a highly effective method for modeling lanthanide-containing systems with reduced computational costs. ${ }^{15-16}$ Furthermore, the relativistic effects in heavy elements can be built into the pseudopotential parameterization. For condensed phase codes, pseudopotentials are a requisite, often accompanied by large plane wave basis sets required to model core electrons.

Several quantum chemistry groups have developed lanthanide pseudopotentials that are reported in the literature. Examples include: (i) Dolg et al., derived energy-consistent small-core and f-in-core pseudopotentials for lanthanides. ${ }^{15-18}$ (ii) Ross et al., developed norm-conserving pseudopotentials with 54 core electrons. ${ }^{19}$ (iii) Cundari et al., proposed that 46 -electron core pseudopotentials provide the best compromise between computational savings and chemical accuracy. ${ }^{20}$ (iv) Hay and Wadt report a 54core electron pseudopotential for lanthanum. ${ }^{21}$ Although the mentioned pseudopotentials have been widely used by the quantum chemical community, most electronic structure calculations with lanthanides include less than $\sim 100$ atoms. ${ }^{22-23}$ Besides gas phase calculations, lanthanides in solution have been modeled with the first solvent shell explicitly ${ }^{24-27}$, and solid-state calculations with lanthanides ${ }^{28}$ and actinides ${ }^{29}$ have been performed as well. Modeling lanthanides in the solid or condensed phases requires plane waves and pseudopotentials that, in principle, enable large scale calculations (i.e., $10^{2}-10^{3}$ atoms with periodic boundary conditions, with full explicit solvent boxes and/or in the condensed phase), and molecular sampling from ab initio molecular dynamics (AIMD), which can be used to determine physical and chemical properties of lanthanide-containing systems. ${ }^{13,30}$ Bachelet, Hamann, and Schüler were the first to publish a set of norm-conserving pseudopotentials for all elements up to $\mathrm{Pu},{ }^{31}$ later followed by Harwigsen, Goedecker and Hutter. ${ }^{32-33}$ 
In the late 90s, Goedecker, Teter and Hutter (GTH) proposed a dual-space Gaussian-type pseudopotential that is separable and satisfies a quadratic scaling with respect to system size. ${ }^{32-33}$ The employment of GTH pseudopotentials in a mixed Gaussian-planewave scheme has proven to be an effective and efficient way to perform AIMD simulations. ${ }^{34}$ Accurate GTH pseudopotentials are available for most elements, ${ }^{32-33,35}$ except for lanthanides and actinides due to the way that their $f$ states were included into the fitting procedure of the potentials, which in effect removed their variational flexibility. This problem becomes most acute when dealing with multiple oxidation states, since a change in redox state will induce a change in the electronic structure of the $f$ orbitals. This compromises the transferability of the pseudopotentials and limits their use in many chemical applications.

Recently, cerium pseudopotentials and basis sets were optimized to study the surface properties of ceria. ${ }^{36-37}$ It was demonstrated that a full inclusion of $f$ states into the fitting procedure can result in highly transferable, though computationally expensive, potentials. ${ }^{37}$ However, accurate GTH pseudopotentials and basis sets for the remaining lanthanides are still lacking, preventing larger-scale DFT calculations and AIMD simulations of lanthanide-containing systems in the condensed phase or solid state.

The objective of this work is to fill this critical gap by producing a full set of well benchmarked pseudopotentials for the entire lanthanide series along with the corresponding Gaussian basis sets. Although these same potentials could be used as a starting point for higher levels of approximation to the electronic structure, our goal is to provide a reliable tool for simulating condensed phase systems including bulk solids, surfaces, and molecular species in gas and solution at a gradient corrected DFT level of theory. Hence, we report our optimized GTH pseudopotentials and corresponding basis sets with uncontracted valence $4 f$ states with Gaussian exponents. For the late lanthanides ( $\mathrm{Tb}$ to $\mathrm{Lu}$ ), the $4 d^{10}$ configuration is treated as a semi-core state. Also, as the on-site Coulomb interactions are particularly strong for localized $2 p, 3 d$ and $4 f$ electrons due to the quantum primogenic effect, ${ }^{38}$ we determined the corresponding Hubbard term $(+\mathrm{U})$ values based on the third ionization potential. The accuracy of our lanthanide GTH pseudopotentials and basis sets with uncontracted $f$ states is illustrated by a series of benchmarks on lanthanide molecules in the gas phase and solid-state.

\section{THEORETICAL AND COMPUTATIONAL METHODOLOGY}

\subsection{GTH pseudopotentials and MOLOPT basis sets}

The norm-conserving, separable, dual-space GTH pseudopotentials comprises of two parts. ${ }^{32,35} \mathrm{~A}$ 
local part given by:

$V_{l o c}^{p p}(r)=-\frac{Z_{i o n}}{r} \operatorname{erf}\left(\alpha^{P P} r\right)+\sum_{i}^{4} C_{i}^{p p}\left(\sqrt{2} \alpha^{P P} r\right)^{2 i-2} \times \exp \left[-\left(\alpha^{P P} r\right)^{2}\right]$

where,

$\alpha^{P P}=\frac{1}{\sqrt{2} r_{l o c}^{p p}}$

where $r_{l o c}^{p p}$ is the range of the Gaussian ionic charge distribution.

A non-local part given by:

$V_{n l}^{p p}\left(\boldsymbol{r}, \boldsymbol{r}^{\prime}\right)=\sum_{l m} \sum_{i j}<\boldsymbol{r}\left|p_{i}^{l m}>h_{i j}^{l}<p_{j}^{l m}\right| \boldsymbol{r}^{\prime}>$

in which Gaussian-type projectors are:

$<\boldsymbol{r} \mid p_{i}^{l m}>=N_{i}^{l} Y^{l m}(r) r^{l+2 i-2} \exp \left[-\frac{1}{2}\left(\frac{r}{r_{l}}\right)^{2}\right]$.

where $N_{i}^{l}$ is a normalization constant, and $Y^{l m}$ is a Laplace's spherical harmonic.

These pseudopotentials have optimal decay properties in both real space and Fourier space, which can be calculated analytically, and are critical for condensed phase calculations. ${ }^{32}$ VandeVondele and Hutter ${ }^{39}$ proposed molecularly optimized (MOLOPT) Gaussian basis sets with analytical dualspace GTH pseudopotentials, where all angular-momentum functions share the same exponents in MOLOPT basis sets.

We followed the general procedure shown in Figure 1 to optimize the lanthanide GTH pseudopotentials and corresponding MOLOPT basis sets. Our derivation of lanthanide GTH pseudopotentials closely follows the scheme by Goedecker et al. ${ }^{32-33}$ First, we fitted the pseudopotential parameters to obtain the best representation of orbital eigenvalues and charge density of the atomic ground states. The $f$ states were handled by explicitly fitting the non-local projectors in each step of the pseudopotential optimization. The GTH pseudopotential parameters, for the ubiquitously used PBE functional, were optimized with respect to atomic all-electron wavefunctions, obtained from fully relativistic density functional calculations specificly modified to directly fit the $f$ states, as originally proposed. ${ }^{33}$ For the fitting process, we used a weighted average of Kohn-Sham eigenvalues, radial densities, and location of the radial nodes. The weights of these factors were heaviest on the semi-core states $(5 s, 5 p, 4 d)$, one order of magnitude lower than the valence states $(6 s, 6 d, 5 d, 4 f)$, and a further reduction of one order of magnitude for the virtual states. Typical differences between the optimized orbital enegies of pseudo-wavefunctions and all-electron wavefunctions in the valence region were $\sim 10^{-}$ 
${ }^{4}-10^{-5}$ Hartree, and $\sim 10^{-3}$ Hartree for unoccupied orbitals. Given the large impact of the $r_{\text {loc }}$ parameter on the fitting procedure with respect to all other parameters, we fitted the potentials by first fixing $r_{\text {loc }}$ and relaxing all other parameters, then choosing the best fit from the resulting series of potentials. We uncontracted the $f$ states with additional Gaussian exponents. We previously followed this scheme to produce accurate calculations for cerium. ${ }^{37}$ The contraction coefficients of the $s, p$, and $d$ states were optimized to minimize the objective function, which consists of energies and condition numbers of the overlap matrix of training molecules. The resulting pseudopotentials and basis sets appear in the Supporting Information (SI), as well as a more detailed discussion about the optimization procedures in Part A of the SI, Tables S1-S3.

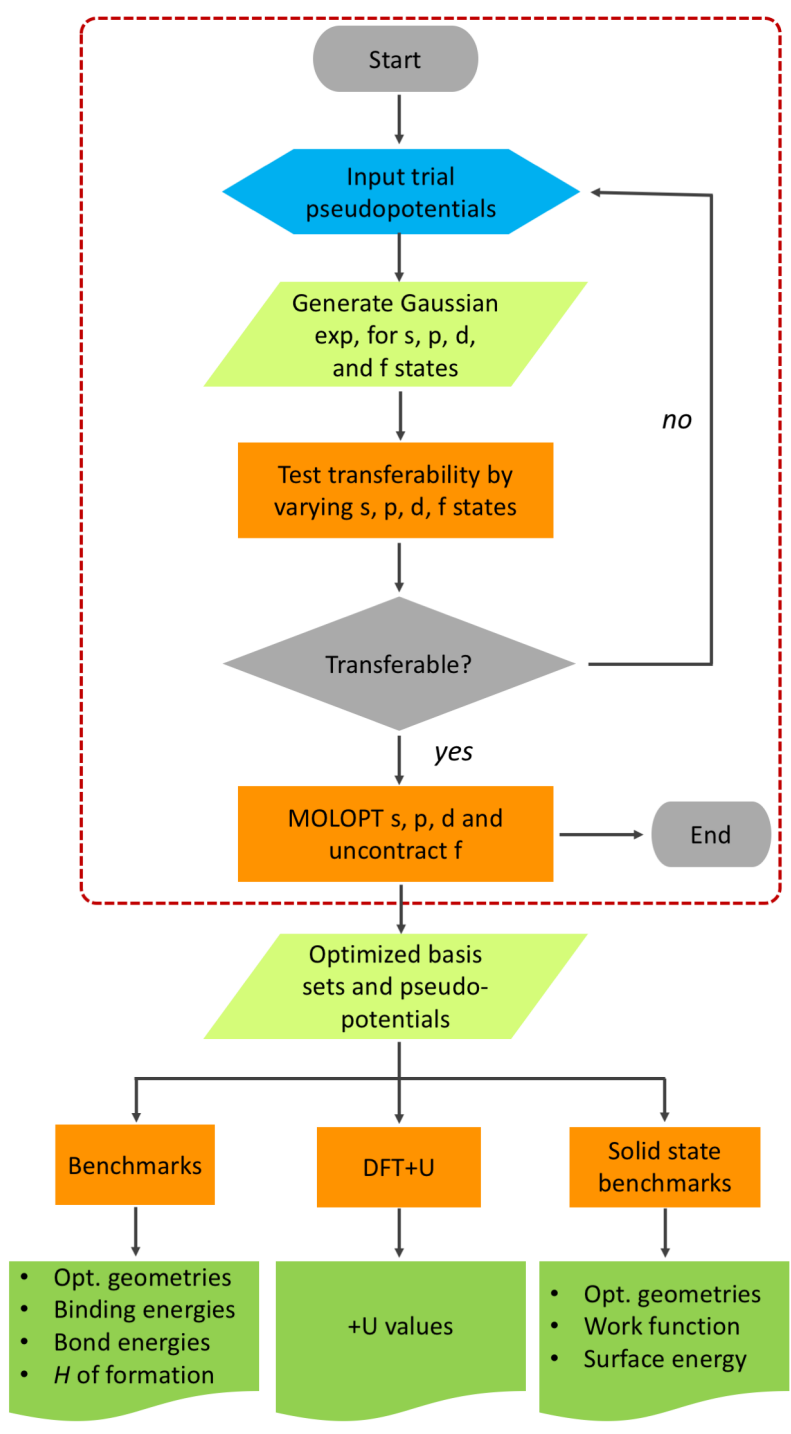

Figure 1. Our general procedure to generate, optimize and benchmark the GTH pseudopotentials and companion basis sets.

\subsection{Computational methodology}


We used CP2K (version 4.1) and CPMD (version 4.1) for benchmarking the optimized pseudopotentials and basis sets. ${ }^{40-43}$ To assess pseudopotential transferability, we calculated two redox reaction energies, and compared results with all-electron calculations performed with the standard quantum chemistry software ADF (version 2016.106) that uses Slater-type basis functions. ${ }^{44}$ Noting that the most stringent test of the transferability of a pseudopotential is its ability to reproduce the energy of redox chemistry, we focused on two prototypical reactions, chosen to represent the most common spin and oxidation states that dominate lanthanide chemistry:

$$
\begin{aligned}
& \mathrm{LnCl}+\frac{1}{2} \mathrm{Cl}_{2}=\mathrm{LnCl}_{2} \\
& \mathrm{LnCl}_{2}+\frac{1}{2} \mathrm{Cl}_{2}=\mathrm{LnCl}_{3}
\end{aligned}
$$

An ionic model of $\mathrm{LnCl}, \mathrm{LnCl}_{2}, \mathrm{LnCl}_{3}$ is assumed where chlorine atoms are treated as closed-shell anions, and the lanthanide including all the spin density with electrons occupying the same orbitals as a lanthanide cation. ${ }^{45}$ The electron configurations of lanthanide ions followed Peterson's work. ${ }^{46}$ The electron configurations of $\mathrm{LnCl}, \mathrm{LnCl}_{2}$ and $\mathrm{LnCl}_{3}$ are depicted in Table S4 of the SI. $\mathrm{LnCl}_{\mathrm{n}}(\mathrm{n}=1-3)$ and $\mathrm{Cl}_{2}$ structures were optimized using Gaussian ${ }^{47}$ (version Gaussian09 D.01) at the $\mathrm{PBE}^{48}$ level. We use the same geometries (calculated with Gaussian) to compare the redox reaction energy for ADF and $\mathrm{CP} 2 \mathrm{~K}$ for consistency. After we get optimized pseudopotential and basis sets, we use the optimized geometries and computed the reaction energetics (Table S11). Relativistic effects were included using the ECP28MWB Ln effective core potentials (ECP).${ }^{16}$ We used the ECP28MWB_SEG basis sets for Ln atoms and cc-pVTZ basis sets for $\mathrm{Cl}^{16,49}$ Minima were confirmed by vibrational analysis that resulted in all positive normal modes of the optimized geometries.

Using these optimized geometries, we performed PBE single-point calculations with the ZORA relativistic correction and TZ2P all-electron basis sets for $\mathrm{Ln}$ and $\mathrm{Cl}^{50-55}$ Three different schemes were tested to compute the reaction energetics of lanthanides, with reactions R1 and R2 as reference points using ADF, see detailed discussion in Part B of the SI, Table S5. Scheme 3 (Fermi-Dirac), which is closer to the smearing algorithm in $\mathrm{CP} 2 \mathrm{~K}$, was also used to calculate $\mathrm{R} 2$ reaction energetics and bond energies of $3 d$ transition metals, see Tables S6 and S7 in the SI. In both cases, mean average deviations are quite small, $0.9 \mathrm{kcal} / \mathrm{mol}$ for reaction energies and $3.8 \mathrm{kcal} / \mathrm{mol}$ for bond energies.

In $\mathrm{CP} 2 \mathrm{~K}$, reaction energies were calculated with molecules placed in cubic boxes with cell lengths 
of $20 \AA$, which allows modeling isolated molecules in a periodic code. A cutoff distance of 800 Ry was chosen, based testing with $\mathrm{Ce}$ and $\mathrm{Tb}$ (Tables S8 - S9 in the SI). The energetics of the two reactions were also calculated in $\mathrm{CPMD}^{42-43}$, with a wavefunction cutoff of $250 \mathrm{Ry}$.

Additional benchmark calculations were performed with $\mathrm{CP} 2 \mathrm{~K}$ to test the new GTH pseudopotentials and basis sets with uncontracted $f$ states for molecules and solids. We used a cutoff of 800 Ry owing to the very tight cores of the resulting potentials which necessitate a large number of plane waves to represent their core densities. Non-periodic conditions were used with molecular tests, except for enthalpies of formation where all calculations were done with uncharged boxes in periodic conditions. Periodic conditions were also used for solid state calculations. Results were compared to relativistic, all-electron reference calculations performed with ADF or to published all-electron calculations, as well as to experimental data when available.

\section{RESULTS AND DISCUSSION}

\subsection{Pseudopotential transferability and reaction energies}

The lanthanide series starts (La) with the ground states of the $4 f$ orbitals empty, and ends ( $\mathrm{Lu}$ ) with them fully filled. The $6 s$ orbital is fully occupied in the ground state configuration for all lanthanides. The $5 d$ orbitals are occupied by one electron for $\mathrm{La}, \mathrm{Ce}, \mathrm{Gd}$, and $\mathrm{Lu}$. The $4 f$ orbitals lie energetically above the $5 s$ and $5 p$ orbitals, and are partially occupied for nearly all lanthanides. Because of increased effective nuclear charge, the $4 f$ orbitals become more and more contracted from La to Lu as shown in Figure $2 \mathrm{~b}$. Generally, for lanthanides, the $4 f, 5 d$ and $6 s$ orbitals are treated as valence orbitals, and the semi-core $5 s$ and $5 p$ orbitals expand into valence region too, as shown in Figure $2 \mathrm{a}$. Therefore, the $5 s$ and $5 p$ orbitals are treated as semi-core states in the "large-core" pseudopotential scheme.

Hartwigsen and co-workers published the first relativistic GTH pseudopotentials for lanthanides, ${ }^{33}$ and their large-core GTH pseudopotentials and MOLOPT basis sets are included in CP2K data library (LnPP0 hereafter). However, the existing LnPP0 pseudopotentials and basis sets do not replicate redox reaction energies, which motivated our work. 


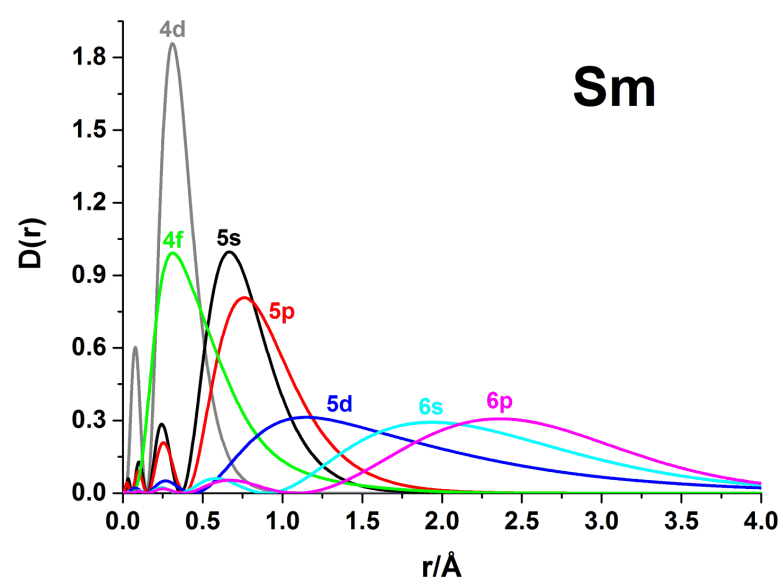

(a)

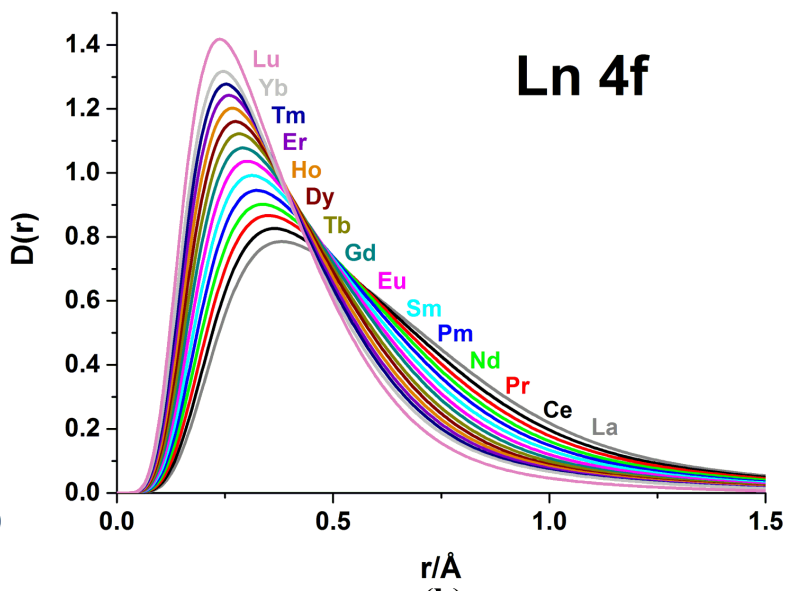

(b)

Figure 2. (a) Radial densities $\mathrm{D}(\mathrm{r})=\mathrm{r}^{2} \mathrm{R}(\mathrm{r})^{2}$ for $4 d, 5 s, 5 p, 4 f, 5 d$ and $6 s$ orbitals of atomic ions $\mathrm{Sm}^{3+}$.

(b) Radial densities $\mathrm{r}^{2} \mathrm{R}(\mathrm{r})^{2}$ of $4 f$ orbitals from $\mathrm{La}^{3+}$ to $\mathrm{Lu}^{3+}$.

To test the transferability of the pseudopotentials and basis sets, we calculated the R1 and R2 reaction energies with lanthanide ions in the $+1,+2$ and +3 oxidation states. Table 1 compares reaction energies obtained with the LnPP0 GTH pseudopotentials with their corresponding ADF results that serve as a comparison point. Reaction energies do not match those computed with ADF when using the basis sets with contracted $s, p, d$ and $f$ states, since results underestimate reaction energies with early lanthanides and overestimate them for the late lanthanides. We uncontracted the $f$ states by using the Gaussian exponents from the CRENBL ECP basis set, ${ }^{19}$ as previously done for cerium by our group. ${ }^{37}$ By using the uncontracted $f$ states, we reduced the error significantly for the early lanthanides (La to $\mathrm{Gd})$. However, the reaction energetics of late lanthanides ( $\mathrm{Tb}$ to $\mathrm{Yb}$ ) are still overestimated compared to the ADF reaction energies (Table 1). Therefore, uncontracting the $f$ states in the LnPP0 basis sets improves their reaction energy accuracy for early lanthanides, but significant errors remain for late lanthanides. 
Table 1. Reaction energies ( $\mathrm{kcal} / \mathrm{mol})$ with previously existing LnPP0 lanthanide pseudopotentials and basis sets at the PBE level. Error calculated with respect to the reaction energies computed with ADF.

\begin{tabular}{ccccccccc}
\hline & \multicolumn{2}{c}{$f$ contracted $^{\mathrm{a}}$} & \multicolumn{2}{c}{$f$ uncontracted $^{\mathrm{b}}$} & \multicolumn{2}{c}{ Contracted error $^{\mathrm{c}}$} & \multicolumn{2}{c}{${\text { Uncontracted } \text { error }^{\mathrm{c}}}$} \\
\cline { 2 - 10 } & $\mathrm{R} 1$ & $\mathrm{R} 2$ & $\mathrm{R} 1$ & $\mathrm{R} 2$ & $\mathrm{R} 1$ & $\mathrm{R} 2$ & $\mathrm{R} 1$ & $\mathrm{R} 2$ \\
\hline $\mathrm{La}$ & -90.3 & -89.3 & -90.3 & -89.3 & 3.9 & 0.8 & 3.9 & 0.8 \\
\hline $\mathrm{Ce}$ & -89.8 & -70.5 & -91.3 & -81.2 & 1.6 & 14.0 & 0.1 & 3.3 \\
\hline $\mathrm{Pr}$ & -83.9 & -56.2 & -85.9 & -73.3 & 3.0 & 20.4 & 0.9 & 3.2 \\
\hline $\mathrm{Nd}$ & -80.4 & -43.0 & -80.3 & -61.0 & -0.8 & 26.8 & -0.6 & 8.7 \\
\hline $\mathrm{Pm}$ & -79.6 & -59.4 & -82.9 & -66.8 & 0.2 & 4.0 & -3.0 & -3.4 \\
\hline $\mathrm{Sm}$ & -82.6 & -28.9 & -81.3 & -37.0 & -5.2 & 19.5 & -3.8 & 11.3 \\
\hline $\mathrm{Eu}$ & -76.7 & -13.4 & -76.6 & -20.3 & -1.7 & 19.9 & -1.5 & 13.1 \\
\hline $\mathrm{Gd}$ & -89.8 & -83.4 & -88.1 & -81.9 & 3.8 & 0.4 & 5.3 & 1.9 \\
\hline $\mathrm{Tb}$ & -73.1 & -95.7 & -73.7 & -93.1 & -3.0 & -13.2 & -3.6 & -10.6 \\
\hline $\mathrm{Dy}$ & -78.6 & -95.6 & -76.7 & -95.5 & -4.4 & -29.5 & -2.5 & -29.4 \\
\hline $\mathrm{Ho}$ & -77.4 & -93.2 & -76.9 & -91.8 & -4.4 & -28.3 & -3.9 & -26.9 \\
\hline $\mathrm{Er}$ & -72.2 & -94.5 & -71.0 & -93.8 & -2.1 & -27.6 & -0.9 & -26.9 \\
\hline $\mathrm{Tm}$ & -75.6 & -75.0 & -74.5 & -81.8 & -3.3 & -22.9 & -2.2 & -29.8 \\
\hline $\mathrm{Yb}$ & -77.6 & -58.2 & -72.8 & -72.2 & -3.7 & -18.8 & 1.1 & -32.7 \\
\hline $\mathrm{Lu}$ & -72.0 & -89.9 & -70.3 & -88.3 & 0.5 & -4.8 & 2.2 & -3.2 \\
\hline
\end{tabular}

As demonstrated by Goedecker and Maschke, pseudopotential transferability is related to the existence of a region around the nuclei where its charge density is practically independent of the chemical environment. ${ }^{56}$ The ideal choice of the cutoff radius $\left(r_{\mathrm{c}}\right)$ is one that distinguishes these two regions. Core electrons should reside exclusively within an inert region, ${ }^{56}$ where charge density does not change in different chemical environments. The significant errors in Table 1 are likely due to the fact that an inert region was not identified in the LnPP0 large-core pseudopotentials. Noticeably, uncontracting the $f$ states did not improve results for dysprosium. Therefore, we generated tighter normconserving GTH pseudopotentials, especially for the late lanthanides where the LnPP0 pseudopotentials are less accurate.

The semi-core state is also important for lanthanide pseudopotentials. Dolg and co-workers found 
that the most accurate pseudopotentials include all orbitals with the same main quantum number as the conventional valence orbitals (e.g., $4 s, 4 p, 4 d, 4 f$ in valence) ${ }^{15-17,57}$ Transferable pseudopotentials have clearly delimited core and valence regions, making it possible to replace the core electrons with a normconserving potential. This is a challenge for the lanthanides, since the $f$ states are close to the core but should be considered as part of the valence, as shown by the spatial overlap between the $4 d$ orbitals and $4 f$ orbitals (Figure 2a). This effect is even more pronounced for the late lanthanides due to contraction (Figure 2b).

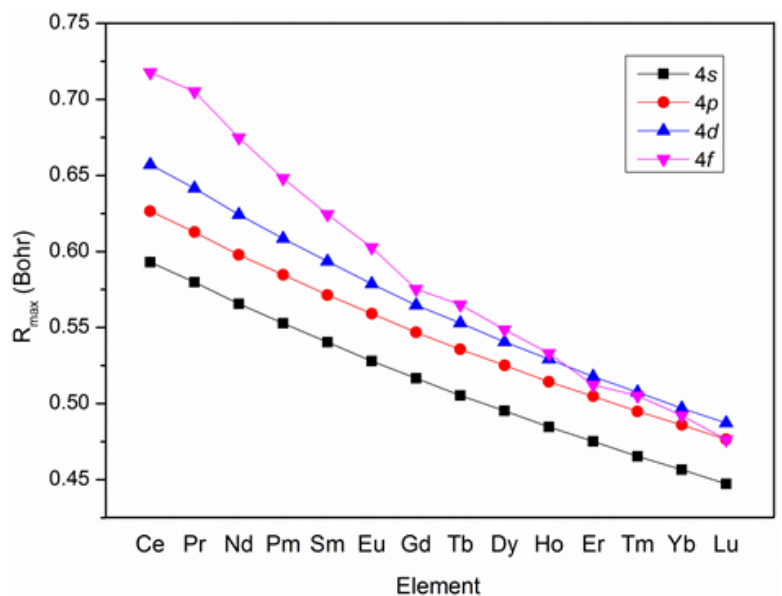

(a)

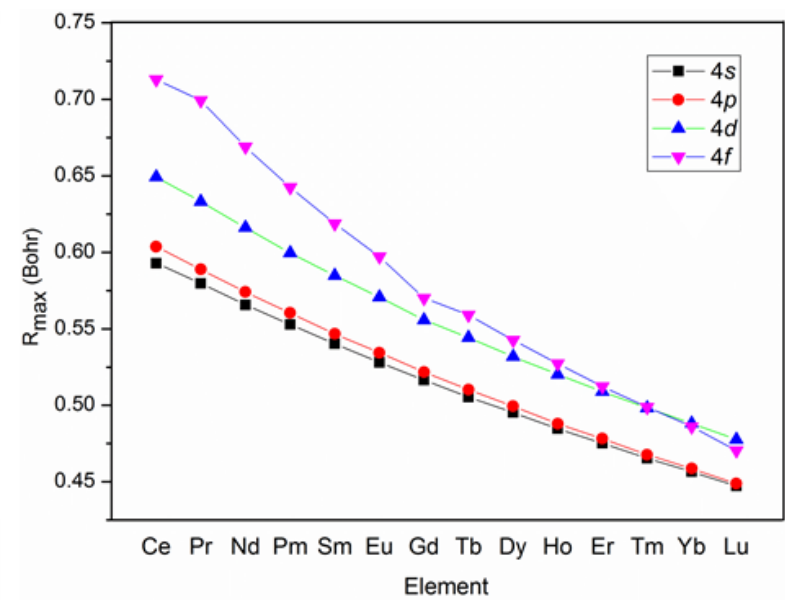

(b)

Figure 3. The $\mathrm{R}_{\max }$ of $4 s, 4 p, 4 d$ and $4 f$ orbitals derived from numerical Dirac-Fock calculations. For (a), $\mathrm{j}=1+\frac{1}{2}$; for $(\mathrm{b}), \mathrm{j}=1-\frac{1}{2}$.

To compare the orbital properties of $4 s, 4 p, 4 d$, and $4 f$ orbitals quantitatively, we obtained the radius of maximum radial densities $\left(\mathrm{R}_{\max }\right)$ of these orbitals (Figure 3 ) with numerical relativistic DiracFock calculations. ${ }^{58} \mathrm{We}$ found no significant boundary between the $4 f$ and $4 d$ orbitals for $\mathrm{Tb}$ to Lu. Therefore, the spatial correlation between the $4 d$ and $4 f$ orbitals is highly relevant due to the compactness of $4 f$ orbitals, as has been discussed by Gomes and co-workers. ${ }^{59}$ The high correlation between $4 d$ and $4 f$ orbitals likely explains the reaction energy results with the LnPP0 large-core lanthanide pseudopotentials (Table 1).

For increased accuracy, the $4 s, 4 p$ and $4 d$ orbitals are usually treated as semi-core states near the valence space. Since pseudopotentials with semi-core wave functions are computationally more expensive, a balance between chemical accuracy and computational cost has to be met. Therefore, we included the more relevant $4 d^{10}$ configuration as a semi-core state, while keeping the $4 s$ and $4 p$ states 
in the core, as there is significant boundary between $4 d$ and $4 s, 4 p$ orbitals (Figure 3). We tested different

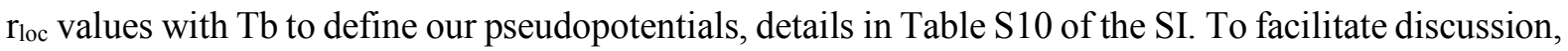
we classified pseudopotentials by their cores (Table 2). As example for Ce, detailed definitions of three different core-region pseudopotentials are depicted in Table 2.

Table 2. The electronic configurations of core, semi-core and valence region for small-core, mediumcore and large-core pseudopotentials for Ce.

\begin{tabular}{cccc}
\hline \hline Core type & Core $^{\mathrm{a}}$ & Semi-core & Valence \\
\hline Small-core & {$[\mathrm{Ar}] 3 \mathrm{~d}^{10}$} & $4 \mathrm{~s}^{2} 4 \mathrm{p}^{6} 4 \mathrm{~d}^{10} 5 \mathrm{~s}^{2} 5 \mathrm{p}^{6}$ & $4 \mathrm{f}^{1} 5 \mathrm{~d}^{1} 6 \mathrm{~s}^{2}$ \\
\hline Medium-core & {$[\mathrm{Kr}]$} & $4 \mathrm{~d}^{10} 5 \mathrm{~s}^{2} 5 \mathrm{p}^{6}$ & $4 \mathrm{f}^{1} 5 \mathrm{~d}^{1} 6 \mathrm{~s}^{2}$ \\
\hline Large-core & {$[\mathrm{Kr}] 4 \mathrm{~d}^{10}$} & $5 \mathrm{~s}^{2} 5 \mathrm{p}^{6}$ & $4 \mathrm{f}^{1} 5 \mathrm{~d}^{1} 6 \mathrm{~s}^{2}$ \\
\hline
\end{tabular}

${ }^{\mathrm{a}}[\mathrm{Ar}]$ and $[\mathrm{Kr}]$ mean the electronic configurations of $\mathrm{Ar}$ and $\mathrm{Kr}$ atoms.

We adopted a large-core pseudopotential scheme for the early lanthanides ( $\mathrm{La}$ to $\mathrm{Gd}$ ), and a medium-core scheme for the late lanthanides ( $\mathrm{Tb}$ to $\mathrm{Lu}$ ). Our new GTH pseudopotentials, and corresponding MOLOPT basis sets with uncontracted $f$ states, are displayed in Part F of the SI.

The energies of the two redox reaction (R1 and R2), computed using our new GTH pseudopotentials and basis sets in both $\mathrm{CP} 2 \mathrm{~K}$ and CPMD, are listed in Table 3. Errors with respect to ADF all-electron calculations are considerably smaller: less than 5\% for all lanthanides except $\mathrm{Nd}$ and Dy. Notably, reaction energies for late lanthanides are not being significantly overestimated, a result that is consistent with our discussion about the overlap between the $4 d$ and $4 f$ orbitals (Figure 3 ). Based on this, we suspect that results for Nd and Dy could be further improved with medium-core and smallcore pseudopotentials, respectively.

Our new GTH pseudopotentials and MOLOPT basis sets with uncontracted $f$ states (LnPP1 hereafter) show accuracy of $2-3 \mathrm{kcal} / \mathrm{mol}$ (mean absolute deviation) when using $\mathrm{CP} 2 \mathrm{~K}$. As a comparison, we also include results with CPMD (Table 3), using planewave basis sets. In principle, results should be converging to the same values at the limit of complete Gaussian or planewave basis sets. However, the ability to control the final electronic state during the initial guess and self-consistent field procedure differs between the two codes, and this control is critical for the electronic structure of the $f$-block elements. Compared with Gaussian basis sets, very large planewave basis sets have difficulty converging to the same states with tightly contracted $4 f$ orbitals, as shown in Table 3 . 
Table 3. Reaction energies (kcal/mol) with our new LnPP1 GTH lanthanide pseudopotentials and MOLOPT basis sets with uncontracted $f$ states, at the PBE level. Mean absolute deviations (MAD) are calculated with respect to the reaction energies calculated with ADF.

\begin{tabular}{ccccccccc}
\hline & \multicolumn{2}{c}{ CP2K } & \multicolumn{2}{c}{ CPMD } & \multicolumn{2}{c}{ CP2K error } & \multicolumn{2}{c}{ CPMD error } \\
\cline { 2 - 9 } & $\mathrm{R} 1$ & $\mathrm{R} 2$ & $\mathrm{R} 1$ & $\mathrm{R} 2$ & $\mathrm{R} 1$ & $\mathrm{R} 2$ & $\mathrm{R} 1$ & $\mathrm{R} 2$ \\
\hline $\mathrm{La}$ & -89.7 & -88.6 & -85.7 & -50.9 & 4.4 & 1.5 & 8.4 & 39.3 \\
\hline $\mathrm{Ce}$ & -88.5 & -85.4 & -76.9 & -63.8 & 2.9 & -0.9 & 14.5 & 20.7 \\
\hline $\mathrm{Pr}$ & -85.7 & -73.8 & -76.9 & -46.3 & 1.1 & 2.8 & 9.9 & 30.3 \\
\hline $\mathrm{Nd}$ & -77.6 & -62.3 & -72.9 & -43.0 & 2.1 & 7.4 & 6.7 & 26.7 \\
\hline $\mathrm{Pm}$ & -80.6 & -61.9 & -77.1 & -53.5 & -0.8 & 1.5 & 2.7 & 9.9 \\
\hline $\mathrm{Sm}$ & -78.9 & -48.4 & -72.7 & -29.2 & -1.4 & -0.0 & 4.7 & 19.1 \\
\hline $\mathrm{Eu}$ & -75.9 & -32.3 & -72.0 & -8.1 & -0.9 & 1.0 & 3.0 & 25.2 \\
\hline $\mathrm{Gd}$ & -89.3 & -81.3 & -87.4 & -80.8 & 4.2 & 2.5 & 6.1 & 3.0 \\
\hline $\mathrm{Tb}$ & -68.4 & -83.6 & -69.9 & -92.6 & 1.7 & -1.0 & 0.2 & -10.0 \\
\hline $\mathrm{Dy}$ & -76.4 & -60.9 & -70.7 & -24.7 & -2.1 & 5.3 & 3.5 & 41.5 \\
\hline $\mathrm{Ho}$ & -71.8 & -65.3 & -72.3 & -74.1 & 1.3 & -0.4 & 0.8 & -9.1 \\
\hline $\mathrm{Er}$ & -67.6 & -68.6 & -78.0 & -82.7 & 2.5 & -1.7 & -7.9 & -15.8 \\
\hline $\mathrm{Tm}$ & -74.0 & -50.1 & -68.7 & -62.9 & -1.7 & 2.0 & 3.6 & -10.8 \\
\hline $\mathrm{Yb}$ & -71.6 & -41.0 & -67.7 & -45.8 & 2.2 & -1.6 & 6.1 & -6.4 \\
\hline $\mathrm{Lu}$ & -67.7 & -87.2 & -72.5 & -83.3 & 4.8 & -2.1 & -0.1 & 1.8 \\
\hline & & & & & $\mathbf{2 . 3}$ & $\mathbf{2 . 1}$ & $\mathbf{5 . 2}$ & $\mathbf{1 8 . 0}$ \\
$\mathrm{MAD}$ & & & & & $\mathbf{k c a l} / \mathbf{m o}$ & $\mathbf{k c a l} / \mathbf{m o}$ & $\mathbf{k c a l} / \mathbf{m o}$ & $\mathbf{k c a l} / \mathbf{m o}$ \\
\hline & & & & $\mathbf{l}$ & $\mathbf{l}$ & $\mathbf{l}$ & $\mathbf{l}$ \\
\hline
\end{tabular}

\subsection{Molecular tests}

We performed tests on molecules in the gas phase to check the accuracy of our new LnPP1 GTH pseudopotentials and companion MOLOPT basis sets. These include lanthanide aqua-ions $\left[\mathrm{Ln}\left(\mathrm{H}_{2} \mathrm{O}\right)_{n}\right]^{3+}$, lanthanide chloride geometries, Ln-Cl bond energies, as well as enthalpies of formation of lanthanide chlorides, fluorides and LnO. Results show small errors compared to experiment and all-electron methods.

The geometry optimization results of lanthanide aqua-ions $\left[\operatorname{Ln}\left(\mathrm{H}_{2} \mathrm{O}\right)_{\mathrm{n}}\right]^{3+},(\mathrm{Ln}=\mathrm{La}-\mathrm{Lu}, \mathrm{n}=8,9)$ are presented in Table 4 and Figure 4. The coordination number $(\mathrm{CN})$ represents the number of water molecules in the first coordination sphere of the lanthanide ion (Table 4). A CN of nine was observed for $\mathrm{La}$ to $\mathrm{Sm}$, and a $\mathrm{CN}$ of eight for $\mathrm{Eu}$ to $\mathrm{Lu}$, in agreement with the reported values for $\mathrm{La}$ and $\mathrm{Lu}^{24}$ Average Ln-O bond lengths calculated with our new pseudopotentials and basis sets are consistent with previous findings $\mathrm{s}^{24,26-27,60}$, except for slightly larger bond lengths $(\sim 1 \%$ for the early lanthanides and 
$\sim 2 \%$ for the late lanthanides, Table 4, Figure 4). The lanthanide contraction quantified as the difference between the average $\mathrm{Ln}-\mathrm{O}$ bond lengths of $\mathrm{La}\left(\mathrm{H}_{2} \mathrm{O}\right)_{9}{ }^{3+}$ and $\mathrm{Lu}\left(\mathrm{H}_{2} \mathrm{O}\right)_{8}{ }^{3+}$, is $0.210 \AA$. This result is in excellent agreement with published electronic structure calculations using four-component relativistic Hartree-Fock (0.210 $\AA)$, MP2 (0.210 $)$, and small-core pseudopotential (0.207 $)^{24,61}$

Table 4. Average Ln-O bond lengths $(\AA)$ and binding energies $(\mathrm{kcal} / \mathrm{mol})$ of lanthanide aqua-ions $\left(\mathrm{Ln}\left(\mathrm{H}_{2} \mathrm{O}\right)_{\mathrm{n}}{ }^{3+}, \mathrm{Ln}=\mathrm{La}-\mathrm{Lu}, \mathrm{n}=8,9\right)$.

\begin{tabular}{|c|c|c|c|c|c|c|c|}
\hline & \multirow{2}{*}{$\mathrm{CN}$} & \multicolumn{2}{|c|}{ Bond Length } & \multicolumn{2}{|c|}{ Binding Energy } & \multicolumn{2}{|c|}{ Error } \\
\hline & & $\mathrm{ADF}$ & $\mathrm{CP} 2 \mathrm{~K}$ & $\mathrm{ADF}$ & $\mathrm{CP} 2 \mathrm{~K}$ & Bond Length $^{\mathrm{a}}$ & Binding Energy \\
\hline $\mathrm{La}$ & 9 & 2.59 & 2.62 & -57.2 & -58.2 & 0.03 & -1.0 \\
\hline $\mathrm{Ce}$ & 9 & 2.57 & 2.59 & -59.3 & -59.7 & 0.02 & -0.4 \\
\hline $\operatorname{Pr}$ & 9 & 2.54 & 2.57 & -61.3 & -61.1 & 0.03 & 0.2 \\
\hline $\mathrm{Nd}$ & 9 & 2.53 & 2.55 & -63.2 & -62.4 & 0.02 & 0.8 \\
\hline $\mathrm{Pm}$ & 9 & 2.52 & 2.54 & -64.0 & -62.8 & 0.02 & 1.2 \\
\hline $\mathrm{Sm}$ & 9 & 2.51 & 2.53 & -63.5 & -63.4 & 0.02 & 0.1 \\
\hline $\mathrm{Eu}$ & 8 & 2.45 & 2.50 & -67.8 & -68.5 & 0.05 & -0.7 \\
\hline $\mathrm{Gd}$ & 8 & 2.43 & 2.46 & -67.4 & -67.6 & 0.03 & -0.2 \\
\hline $\mathrm{Tb}$ & 8 & 2.42 & 2.47 & -69.4 & -68.1 & 0.05 & 1.3 \\
\hline Dy & 8 & 2.41 & 2.46 & -71.4 & -68.0 & 0.05 & 3.4 \\
\hline Ho & 8 & 2.39 & 2.44 & -73.4 & -68.2 & 0.05 & 5.2 \\
\hline $\mathrm{Er}$ & 8 & 2.38 & 2.44 & -74.4 & -69.2 & 0.06 & 5.2 \\
\hline $\mathrm{Tm}$ & 8 & 2.38 & 2.43 & -73.4 & -69.4 & 0.05 & 4.0 \\
\hline $\mathrm{Yb}$ & 8 & 2.37 & 2.43 & -73.1 & -70.3 & 0.06 & 2.8 \\
\hline $\mathrm{Lu}$ & 8 & 2.35 & 2.41 & -72.7 & -71.1 & 0.06 & 1.6 \\
\hline MAD & & & & & & $0.04 \AA$ & $1.9 \mathrm{kcal} / \mathrm{mol}$ \\
\hline
\end{tabular}

a. Error of bond length is calculated by $\mathrm{L}_{\mathrm{CP} 2 \mathrm{~K}}-\mathrm{L}_{\mathrm{ADF}}$, where $\mathrm{L}$ is bond length.

b. Error of binding energy is calculated by $\mathrm{E}_{\mathrm{CP} 2 \mathrm{~K}}-\mathrm{E}_{\mathrm{ADF}}$, where $\mathrm{E}$ is binding energy. 


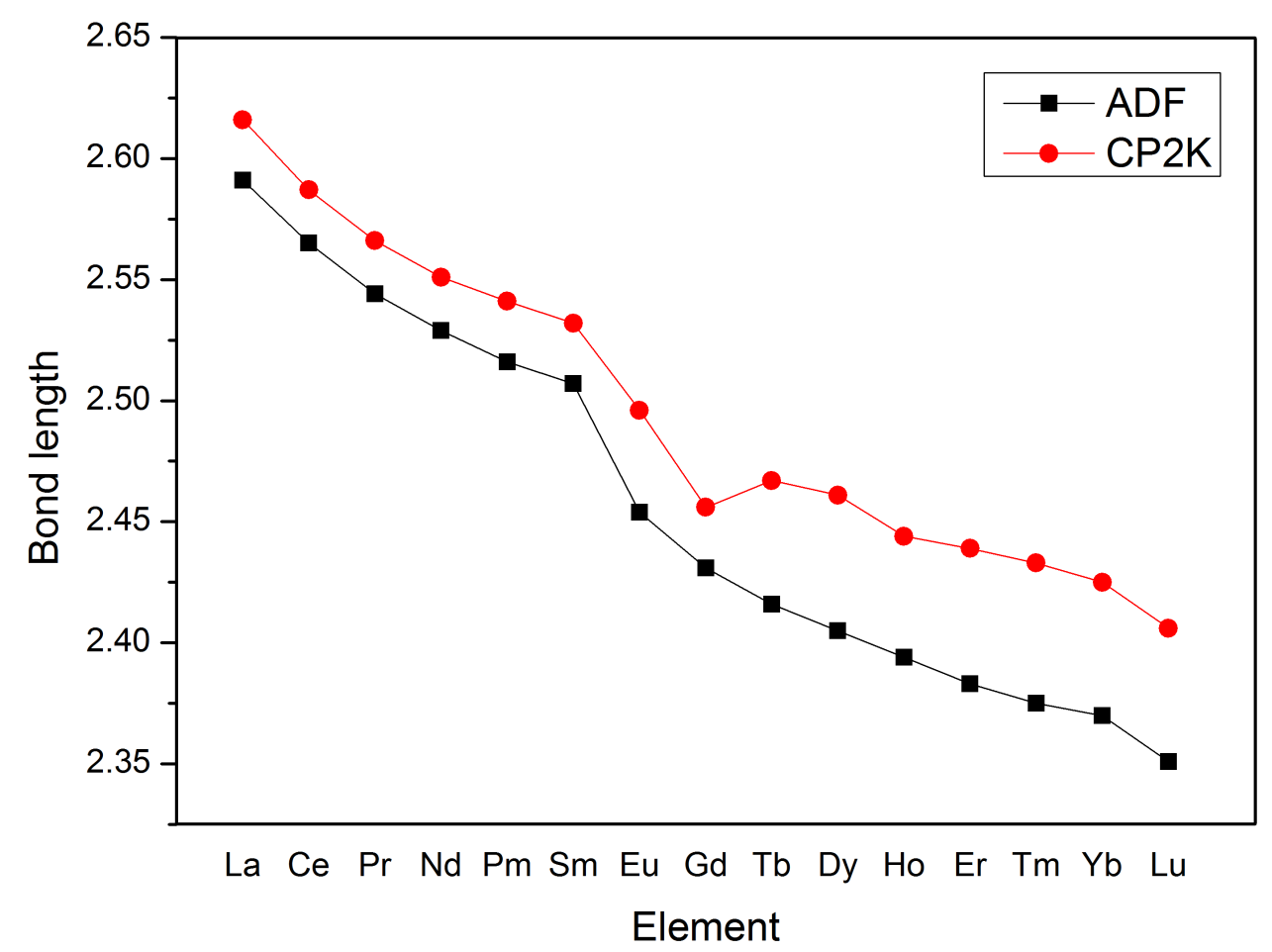

Figure 4. Average Ln-O bond lengths in $\mathrm{Ln}\left(\mathrm{H}_{2} \mathrm{O}\right)_{\mathrm{n}}{ }^{3+}(\mathrm{Ln}=\mathrm{La}-\mathrm{Lu}, \mathrm{n}=8,9)$.

Our new LnPP1 pseudopotentials and basis sets also reproduce the structure of lanthanide chloride compounds $\left(\mathrm{LnCl}_{\mathrm{n}}, \mathrm{n}=1-3\right)$, within $0.04 \AA$ for bond lengths and within 2 degrees for bond angles compared to optimized geometries using with all-electron calculations. Tables S12 and S13 in the SI show the average bond lengths and angles of the optimized structures obtained with ADF using allelectron methods and our LnPP1 pseudopotentials and basis sets with CP2K.

From the optimized structures we also calculated the homolytic bond $\mathrm{Ln}-\mathrm{Cl}$ dissociation energies in $\mathrm{LnCl}_{3}(\mathrm{Ln}=\mathrm{La}-\mathrm{Lu})$ using reaction reaction 3:

$$
\mathrm{LnCl}_{3}=\mathrm{LnCl}_{2}+\mathrm{Cl}(R 3)
$$

Reactions 2 and 3 differ by a constant amount representing half the atomization energy of dichlorine $(61.6 \mathrm{kcal} / \mathrm{mol}$, close to the well-known $\mathrm{Cl}-\mathrm{Cl}$ bond energy of $\sim 58.0 \mathrm{kcal} / \mathrm{mol})$ and the relative errors (both reactions with respect to ADF values) is $\sim 4.0 \mathrm{kcal} / \mathrm{mol}$. The $\mathrm{M}-\mathrm{Cl}$ bond dissocitation energy errors with lanthanides are similar to those computed for $3 d$ transition metal chlorides, see Part B of the SI. 
Table 5. $\mathrm{Ln}-\mathrm{Cl}$ bond energies $(\mathrm{kcal} / \mathrm{mol})$ in $\mathrm{LnCl}_{3}(\mathrm{Ln}=\mathrm{La}-\mathrm{Lu})$ calculated with our $\mathrm{LnPP} 1$ pseudopotentials and basis sets (CP2K) and all-electron (ADF), both with the PBE functional.

\begin{tabular}{cccc}
\hline \hline & ADF & CP2K & Error \\
\hline $\mathrm{La}$ & -125.0 & -119.4 & 5.6 \\
\hline $\mathrm{Ce}$ & -119.3 & -116.2 & 3.1 \\
\hline $\mathrm{Pr}$ & -111.4 & -104.6 & 6.8 \\
\hline $\mathrm{Nd}$ & -104.6 & -93.1 & 11.5 \\
\hline $\mathrm{Pm}$ & -98.2 & -92.7 & 5.5 \\
\hline $\mathrm{Sm}$ & -83.1 & -79.2 & 3.9 \\
\hline $\mathrm{Eu}$ & -68.1 & -63.1 & 5.0 \\
\hline $\mathrm{Gd}$ & -118.6 & -112.1 & 6.5 \\
\hline $\mathrm{Tb}$ & -117.4 & -114.4 & 3.0 \\
\hline $\mathrm{Dy}$ & -101.0 & -91.7 & 9.3 \\
\hline $\mathrm{Ho}$ & -99.8 & -96.1 & 3.7 \\
\hline $\mathrm{Er}$ & -101.7 & -99.4 & 2.3 \\
\hline $\mathrm{Tm}$ & -86.9 & -80.9 & 6.0 \\
\hline $\mathrm{Yb}$ & -74.3 & -71.8 & 2.5 \\
\hline $\mathrm{Lu}$ & -120.0 & -118.0 & 2.0 \\
\hline $\mathrm{MAD}$ & & & $\mathbf{5 . 1} \mathbf{~ k c a l} / \mathbf{m o l}$ \\
\hline
\end{tabular}

We calculated the formation enthalpies of $\operatorname{LnCl}_{n}(n=2,3), \operatorname{LnF}_{n}(n=1,2,3)$, and $\mathrm{LnO}$, which are part of the LnHF54 data set compiled in 2016 by Grimmel et al., where they performed all-electron calculations on the enthalpies of formations with many functionals, including $\mathrm{PBE} .{ }^{45}$ Results show that the MADs of our LnPP1 pseudopotentials and basis sets with respect to experiment are very similar to those reported by Grimmel et al., using the PBE functional. Only the lanthanide molecules with known experimental enthalpies of formation were calculated. Except for the Ln oxidation state of +1 (LnF), our calculated results have similar accuracy to all-electron methods. It should be noted that the allelectron calculations performed by Grimmel et al., are based on single molecules as a gas phase reference, and our $\mathrm{CP} 2 \mathrm{~K}$ calculations were performed under periodic conditions with uncharged boxes and include an empirical fit to experiment, with predictive value throughout the lanthanide series, see Part D of the SI for details, where Tables (S14 to S19) with all the computed enthalpies of formation are reported as well. 
Table 6. Computed MAD values for enthalpies of formation using CP2K with our LnPP1 pseudopotentials and basis sets with respect to experiment, and previously done with all-electron ${ }^{45}$ with respect to experiment. Both methods used the PBE functional.

\begin{tabular}{ccc}
\hline \hline & $\begin{array}{c}\text { CP2K - Experiment } \\
\text { MAD }(\mathrm{kcal} / \mathrm{mol})\end{array}$ & $\begin{array}{c}\text { All-electron - Experiment } \\
\text { MAD }(\mathrm{kcal} / \mathrm{mol})\end{array}$ \\
\hline $\mathrm{LnCl}_{3}$ & 7.8 & 9.6 \\
\hline $\mathrm{LnCl}_{2}$ & 18.6 & 8.0 \\
\hline $\mathrm{LnF}_{3}$ & 28.1 & 34.4 \\
\hline $\mathrm{LnF}_{2}$ & 3.6 & 11.9 \\
\hline $\mathrm{LnF}$ & 33.8 & 4.7 \\
\hline $\mathrm{LnO}$ & 6.1 & 23.0 \\
\hline
\end{tabular}

\subsection{Solid state tests}

Considering the importance of lanthanides in materials science, we tested our LnPP1 pseudopotentials and basis sets in the solid state with lanthanide bulk metals. As calculating solid metals is computationally expensive, we limited our tests to $\mathrm{La}, \mathrm{Eu}, \mathrm{Gd}$ and $\mathrm{Er}$ for which there are experimental data. The calculated lanthanide metal crystal lattice constants are listed in Table 7, and the work functions and surface energies of the (001) surface, computed using slabs made up with of $5 \times 5 \times 3$ layers in periodic conditions, are reported in Table 8 . We calculated work functions as $V_{H}-\varepsilon_{F}$, where $\mathrm{V}_{\mathrm{H}}$ is the one-particle Hartree potential away from the surface and $\varepsilon_{\mathrm{F}}$ is Fermi level of the lanthanide surface. The surface energies were computed as:

$$
\sigma=\frac{E_{\text {slab }}-N \delta E_{\text {bulk }}}{2 A}
$$

in which $E_{\text {slab }}$ is is the energy of the slab, $\delta E_{\text {bulk }}$ is the enegy of each atom in the bulk, $N$ is the number of atoms in the slab, and $2 \mathrm{~A}$ is the total surface area on both sides of the slab.

Fortunately, theoretical and experimental data on lanthanide surface energies and work functions are known. ${ }^{62-69}$ The calculated crystal lattice constants are within $\sim 0.12 \AA$ of experiment (Table 7). The work functions calculated with our pseudopotentials and basis sets have a mean absolute deviation of $0.12 \mathrm{eV}(2.77 \mathrm{kcal} / \mathrm{mol})$ with respect to experimental values, and the surface energies calculated have a mean absolute deviation of $0.21 \mathrm{~J} / \mathrm{m}^{2}$ from experiment, as shown in Table 8 . The values calculated with our LnPP1 GTH pseudopotential and basis sets are also comparable to published theoretical results, see 
Table 8.

Table 7. Crystal lattice constants $(\AA)$ calculated with our LnPP1 GTH pseudopotentials and basis sets with uncontracted $f$ states, at the PBE level.

\begin{tabular}{cccccccc}
\hline \hline & \multirow{2}{*}{$\begin{array}{c}\text { Crystal } \\
\text { Structure }\end{array}$} & \multicolumn{2}{c}{ Geometry (CP2K) } & \multicolumn{2}{c}{ Geometry (Expt.) } & \multicolumn{2}{c}{ Error $^{\mathrm{b}}$} \\
\cline { 3 - 8 } & $\mathrm{a}_{0}$ & $\mathrm{c}_{0}$ & $\mathrm{a}_{0}$ & $\mathrm{c}_{0}$ & $\mathrm{a}_{0}$ & $\mathrm{c}_{0}$ \\
\hline $\mathrm{La}$ & $\mathrm{Hcp}$ & 3.89 & 6.05 & 3.75 & 6.07 & 0.14 & 0.02 \\
\hline $\mathrm{Eu}$ & $\mathrm{Bcc}$ & 4.37 & 4.48 & 4.61 & 4.61 & -0.24 & -0.13 \\
\hline $\mathrm{Gd}$ & $\mathrm{Hcp}$ & 3.74 & 5.99 & 3.63 & 5.78 & 0.11 & 0.21 \\
\hline $\mathrm{Er}$ & $\mathrm{Hcp}$ & 3.59 & 5.66 & 3.56 & 5.59 & 0.03 & 0.07 \\
\hline MAD & & & & & & $\mathbf{0 . 1 3} \AA$ & $\mathbf{0 . 1 1} \AA$ \\
\hline
\end{tabular}

${ }^{a}$ Experiment data reference ${ }^{70}$

b. Error of crystal lattice constant is calculated by $d_{\mathrm{CP} 2 \mathrm{~K}}-\mathrm{d}_{\mathrm{Expt}}$ where $\mathrm{d}$ is crystal lattice constant.

Table 8. Work functions $(\mathrm{eV})$ and surface energies $\left(\mathrm{J} / \mathrm{m}^{2}\right)$ calculated with our new GTH pseudopotentials and basis sets with uncontracted $f$ states, at the PBE level.

\begin{tabular}{cccccccccc}
\hline & \multirow{2}{*}{ Surface } & \multicolumn{4}{c}{ Work function } & \multicolumn{3}{c}{ Surface energy } \\
\cline { 3 - 10 } & & CP2K & Expt. & Theor. & Error $^{\mathrm{a}}$ & CP2K & Expt. & Theor. & Error $^{\mathrm{b}}$ \\
\hline $\mathrm{La}$ & $\mathrm{hcp}(001)$ & 3.19 & $3.50^{\mathrm{c}}$ & $3.21^{\mathrm{d}}$ & -0.31 & 0.75 & $1.02^{\mathrm{g}}$ & $0.57^{\mathrm{h}}$ & -0.27 \\
\hline $\mathrm{Eu}$ & $\mathrm{bcc}(001)$ & 2.52 & $2.50^{\mathrm{c}}$ & $2.42^{\mathrm{d}}$ & 0.02 & 0.31 & $0.45^{\mathrm{g}}$ & $0.34^{\mathrm{h}}$ & -0.14 \\
\hline $\mathrm{Gd}$ & $\mathrm{hcp}(001)$ & 3.07 & $3.10^{\mathrm{e}}$ & $3.30^{\mathrm{f}}$ & 0.03 & 0.91 & - & - & - \\
\hline $\mathrm{Er}$ & $\mathrm{hcp}(001)$ & 3.29 & - & - & - & 1.07 & - & - & - \\
\hline \multirow{2}{*}{ MAD } & & & & & $\mathbf{0 . 1 2}$ & & & & $\mathbf{0 . 2 1}$ \\
& & & & & $\mathbf{e V}$ & & & & $\mathbf{J} / \mathbf{m}^{2}$ \\
\hline
\end{tabular}

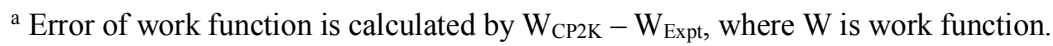

${ }^{b}$ Error of surface energy is calculated by $\mathrm{E}_{\mathrm{CP} 2 \mathrm{~K}}-\mathrm{E}_{\mathrm{Expt}}$, where $\mathrm{E}$ is surface energy.

${ }^{c}$ The experimental data of work function for $\mathrm{La}, \mathrm{Eu}$ is from reference ${ }^{64}$

${ }^{d}$ The previous theoretical data of La, Eu work functions are from reference ${ }^{68}$, where the bcc(110) surface of Eu is computed.

${ }^{\mathrm{e}}$ The experimental data of work function for Gd is from reference ${ }^{63}$

${ }^{\mathrm{f}}$ The previous theoretical data of work function for $\mathrm{Gd}$ is from reference ${ }^{66}$

$\mathrm{g}$ The experimental data of surface energy for $\mathrm{La}, \mathrm{Eu}$ is from reference ${ }^{67}$

${ }^{h}$ The previous theoretical data of surface energy for La, Eu is from reference ${ }^{68}$

\subsection{DFT +U correction}

We performed DFT $+\mathrm{U}$ calculations with our LnPP1 pseudopotentials and basis sets based on third ionization potentials, whose values for lanthanides are known from experiment. ${ }^{71} \mathrm{We}$ varied the $+\mathrm{U}$ 
values to best match experiment (Table 9), details for Ce are shown in Table S20 in the SI. We previously found that, for lanthanides, $U$ values have a larger effect on energies of reaction that involve a change in population of the $4 f$ electrons. ${ }^{37}$ Therefore, no values were calculated for Gd and Lu. Results show that larger $U$ values are required for the early than for the late lanthanides to accurately reproduce ionization potentials. Finally, it is noted that $\mathrm{U}$ values will vary for different properties and need to be fitted accordingly, but this set may serve as a starting point for additional studies, see SI Table S21.

Table 9. The $\mathrm{U}$ value $(\mathrm{eV})$ and third ionization potential $(\mathrm{kcal} / \mathrm{mol})$ calculated by $\mathrm{DFT}$ and $\mathrm{DFT}+\mathrm{U}$, using our LnPP1 GTH pseudopotentials and basis sets with uncontracted $f$ states in CP2K.

\begin{tabular}{|c|c|c|c|c|c|c|}
\hline & $\mathrm{U}$ & DFT & $\mathrm{DFT}+\mathrm{U}$ & Expt. & Error $(\mathrm{DFT})^{\mathrm{a}}$ & Error $(\mathrm{DFT}+\mathrm{U})^{\mathrm{b}}$ \\
\hline $\mathrm{La}$ & 3.27 & 449.7 & 442.4 & 442.2 & 7.5 & 0.2 \\
\hline $\mathrm{Ce}$ & 4.08 & 504.1 & 466.3 & 465.8 & 38.3 & 0.5 \\
\hline $\operatorname{Pr}$ & 4.63 & 528.8 & 498.4 & 498.7 & 30.1 & -0.3 \\
\hline $\mathrm{Nd}$ & 3.54 & 552.5 & 510.6 & 510.6 & 41.9 & 0 \\
\hline $\mathrm{Pm}$ & 5.44 & 552.3 & 514.7 & 514.0 & 38.3 & 0.7 \\
\hline $\mathrm{Sm}$ & 3.54 & 570.0 & 540.9 & 540.3 & 29.7 & 0.6 \\
\hline $\mathrm{Eu}$ & 4.08 & 604.6 & 575.0 & 574.7 & 29.9 & 0.3 \\
\hline $\mathrm{Tb}$ & 2.45 & 527.3 & 506.1 & 505.3 & 22 & 0.8 \\
\hline Dy & 1.90 & 551.0 & 529.5 & 528.8 & 22.2 & 0.7 \\
\hline Ho & 0.54 & 532.0 & 525.8 & 526.7 & 5.3 & -0.9 \\
\hline Er & 1.63 & 541.7 & 525.0 & 524.4 & 17.3 & 0.6 \\
\hline $\mathrm{Tm}$ & 2.18 & 565.5 & 546.0 & 546.1 & 19.4 & -0.1 \\
\hline $\mathrm{Yb}$ & 0.54 & 582.0 & 577.2 & 577.7 & 4.3 & -0.5 \\
\hline MAD & & & & & $23.6 \mathrm{kcal} / \mathrm{mol}$ & $0.5 \mathrm{kcal} / \mathrm{mol}$ \\
\hline
\end{tabular}

\section{CONCLUSIONS}

We have constructed new sets of GTH pseudopotentials and companion basis sets for the whole lanthanide series. We adopted large-core pseudopotentials for lanthanum to gadolinium, and mediumcore pseudopotentials for terbium to lutetium. This scheme provides a good compromise between computational cost and chemical accuracy. The corresponding MOLOPT basis sets were optimized with the $f$ states uncontracted in the valence orbitals. Our LnPP1 GTH pseudopotentials and basis sets performed comparably to all-electron calculations in a variety of molecular and solid-state benchmarks 
that included structural, electronic, and thermodynamic quantities. Additionally, DFT $+\mathrm{U}$ parameters, based on the ionization potentials from experiment, were determined. These new pseudopotentials and basis sets will facilitate larger-scale DFT calculations and AIMD simulations of lanthanide-containing systems in the condensed phase and/or the solid state, where reliable potentials accounting for the chemistry were largely absent. Although this set is based on the PBE functional, it can serve as a starting point for additional parametrization suitable for other functionals, including meta-GGA and hybrid functionals.

\section{SUPPORTING INFORMATION}

The SI is divided into parts corresponding to sections of the main text. Part A has the supporting information on how the pseudopotentials and basis sets were optimized (Section 2.1), along with Tables S1 - S3. Part B has the supporting information on the computational methodology (Section 2.2) with additional detail on the ADF calculations along with Tables S4 to S9. Part C has the supporting information regarding our results with pseudopotential transferability and reaction energies (Section 3.1) with Table S10 and S11. Part D has the supporting information for our molecule test results (Section 3.2) with lanthanide chloride geometries (Figure S1, Tables S12 and S13) and a discussion on the calculation of enthalpies of formation with CP2K (Figure S1, Tables S14-S19). Part E has the supporting information on the DFT $+\mathrm{U}$ calculations (Table S20-S21). Most importantly, Part F includes our LnPP1 pseudopotentials and basis sets in $\mathrm{CP} 2 \mathrm{~K}$ format, so that the scientific community can readily use them.

\section{ACKNOWLEDGMENTS}

The manuscript was partially authored by battelle Memorial Institute under contract No. DEAC05-76RL01830 with the U.S. Department of Energy, The United States Government retains and the publisher, by accepting the article for publication, acknowledges that the United States Government retains a non-exclusive, paid-up, irrevocable, world-wide license to publish or reproduce the published form of this manuscript, or allow others to do so, for United States Government purposes. V.-A.G. was supported by the U.S. Department of Energy, Basic Energy Sciences, Chemistry, Geochemistry and Biological Sciences Separations Program, and R.R. and M.-T.N. were supported by PNNL Laboratory Directed Research and Development CheMSR Agile Investment. J.-B.L. and J.L. were supported by 
the National Natural Science Foundation of China (Nos. 21433005, 91645203, and 21590792). D.C.C. was supported by Research and Innovation at the University of Nevada, Reno. Calculations were performed at PNNL Research Computing, Tsinghua National Laboratory for Information Science and Technology and the Computational Chemistry Laboratory under Tsinghua Xuetang Talents Program, and University of Nevada, Reno High Performance Computing. J.-B. L. and J. L. acknowledge discussions with Dr. Yang-Gang Wang (Southern University of Science and Technology).

\section{REFERENCES}

1. Parker, D.; Williams, J. A. G., Getting excited about lanthanide complexation chemistry. J. Chem. Soc.-Dalton Trans. 1996, (18), 3613-3628.

2. Parker, D.; Dickins, R. S.; Puschmann, H.; Crossland, C.; Howard, J. A. K., Being excited by lanthanide coordination complexes: Aqua species, chirality, excited-state chemistry, and exchange dynamics. Chem. Rev. 2002, 102 (6), 1977-2010.

3. Bettinelli, M.; Carlos, L. D.; Liu, X. G., Lanthanide-doped upconversion nanoparticles. Phys. Today 2015, 68 (9), 7.

4. de Bettencourt-Dias, A., Lanthanide-based emitting materials in light-emitting diodes. Dalton Trans. 2007, (22), 2229-2241.

5. Eliseeva, S. V.; Bunzli, J. C. G., Lanthanide luminescence for functional materials and biosciences. Chem. Soc. Rev. 2010, 39 (1), 189-227.

6. Binnemans, K., Lanthanide-based luminescent hybrid materials. Chem. Rev. 2009, 109 (9), 42834374.

7. Wang, F.; Liu, X. G., Recent advances in the chemistry of lanthanide-doped upconversion nanocrystals. Chem. Soc. Rev. 2009, 38 (4), 976-989.

8. Woodruff, D. N.; Winpenny, R. E. P.; Layfield, R. A., Lanthanide single-molecule magnets. Chem. Rev. 2013, 113 (7), 5110-5148.

9. Guo, F. S.; Day, B. M.; Chen, Y. C.; Tong, M. L.; Mansikkamaki, A.; Layfield, R. A., Magnetic hysteresis up to 80 kelvin in a dysprosium metallocene single-molecule magnet. Science 2018, 362 (6421), 1400-+.

10. Hulliger, F.; Ott, H. R., Superconducting and magnetic properties of Ba2LanthanideCu3O7-x compounds. Z. Phys. B-Condens. Mat. 1987, 67 (3), 291-298.

11. Giess, E. A.; Sandstrom, R. L.; Gallagher, W. J.; Gupta, A.; Shinde, S. L.; Cook, R. F.; Cooper, E. I.; Osullivan, E. J. M.; Roldan, J. M.; Segmuller, A. P.; Angilello, J., Lanthanide gallate pervskitetypre substartes for epitaxial, high Tc superconducting Ba2YCu3O7-delta films. IBM J. Res. Dev. 1990, 34 (6), 916-926.

12. Caravan, P.; Ellison, J. J.; McMurry, T. J.; Lauffer, R. B., Gadolinium(III) chelates as MRI contrast agents: Structure, dynamics, and applications. Chem. Rev. 1999, 99 (9), 2293-2352.

13. Wang, Y. G.; Mei, D. H.; Glezakou, V. A.; Li, J.; Rousseau, R., Dynamic formation of singleatom catalytic active sites on ceria-supported gold nanoparticles. Nat. Commun. 2015, 6, 8.

14. Hu, A. H.; Guo, J. J.; Pan, H.; Zuo, Z. W., Selective functionalization of methane, ethane, and higher alkanes by cerium photocatalysis. Science 2018, 361 (6403), 668-+. 
15. Dolg, M.; Stoll, H.; Savin, A.; Preuss, H., Energy-adjusted pseudopotentials for the rare-earth elements. Theor. Chim. Acta 1989, 75 (3), 173-194.

16. Dolg, M.; Stoll, H., Pseudopotetntial study of the rare-earth monohydrides, monoxies, and monofluorides. Theor. Chim. Acta 1989, 75 (5), 369-387.

17. Cao, X. Y.; Dolg, M., Valence basis sets for relativistic energy-consistent small-core lanthanide pseudopotentials. J. Chem. Phys. 2001, 115 (16), 7348-7355.

18. Weigand, A.; Cao, X. Y.; Yang, J.; Dolg, M., Quasirelativistic f-in-core pseudopotentials and core-polarization potentials for trivalent actinides and lanthanides: molecular test for trifluorides.

Theor. Chem. Acc. 2010, 126 (3-4), 117-127.

19. Ross, R. B.; Gayen, S.; Ermler, W. C., Ab initio relativistic effective potentials with spin-orbit operations. V. Ce through Lu. J. Chem. Phys. 1994, 100 (11), 8145-8155.

20. Cundari, T. R.; Stevens, W. J., Effective core potential methods for the lanthanides. J. Chem. Phys. 1993, 98 (7), 5555-5565.

21. Hay, P. J.; Wadt, W. R., Ab initio effective core potentials for molecular calculations - potentials for the transition-metal atoms Sc to Hg. J. Chem. Phys. 1985, 82 (1), 270-283.

22. Cao, X. Y.; Zhang, J.; Weissmann, D.; Dolg, M.; Chen, X. B., Accurate quantum chemical modelling of the separation of Eu3+ from $\mathrm{Am} 3+/ \mathrm{Cm} 3+$ by liquid-liquid extraction with Cyanex 272 . Phys. Chem. Chem. Phys. 2015, 17 (32), 20605-20616.

23. Huang, P. W.; Wang, C. Z.; Wu, Q. Y.; Lan, J. H.; Song, G.; Chai, Z. F.; Shi, W. Q., Theoretical studies on the synergistic extraction of Am3+ and Eu3 + with CMPO-HDEHP and CMPO-HEH EHP systems. Dalton Trans. 2018, 47 (15), 5474-5482.

24. Clark, A. E., Density functional and basis set dependence of hydrated Ln(III) properties. J. Chem. Theory Comput. 2008, 4 (5), 708-718.

25. Lan, J. H.; Shi, W. Q.; Yuan, L. Y.; Zhao, Y. L.; Li, J.; Chai, Z. F., Trivalent actinide and lanthanide separations by tetradentate nitrogen ligands: a quantum chemistry study. Inorg. Chem. 2011, 50 (19), 9230-9237.

26. Zhang, J.; Heinz, N.; Dolg, M., Understanding lanthanoid(III) hydration structure and kinetics by insights from energies and wave functions. Inorg. Chem. 2014, 53 (14), 7700-7708.

27. Zhang, J.; Dolg, M., Labile capping bonds in lanthanide(III) complexes: shorter and weaker. $J$. Phys. Chem. A 2015, 119 (4), 774-780.

28. Hulsen, M.; Dolg, M.; Link, P.; Ruschewitz, U., Improved valence basis sets for divalent lanthanide 4f-in-core pseudopotentials. Theor. Chem. Acc. 2011, 129 (3-5), 367-379.

29. Kudin, K. N.; Scuseria, G. E.; Martin, R. L., Hybrid density-functional theory and the insulating gap of UO2. Phys. Rev. Lett. 2002, 89 (26), 4.

30. Li, X.; Samin, A.; Zhang, J.; Unal, C.; Mariani, R. D., Ab initio molecular dynamics study of lanthanides in liquid sodium. J. Nucl. Mater. 2017, 484, 98-102.

31. Bachelet, G. B.; Hamann, D. R.; Schluter, M., Pseudopotentials that work - from H to Pu. Phys.

Rev. B 1982, 26 (8), 4199-4228.

32. Goedecker, S.; Teter, M.; Hutter, J., Separable dual-space Gaussian pseudopotentials. Phys. Rev. $B$ 1996, 54 (3), 1703-1710.

33. Hartwigsen, C.; Goedecker, S.; Hutter, J., Relativistic separable dual-space Gaussian pseudopotentials from H to Rn. Phys. Rev. B 1998, 58 (7), 3641-3662.

34. Lippert, G.; Hutter, J.; Parrinello, M., A hybrid gaussian and plane wave density functional scheme. Molecular Physics 1997, 92 (3), 477-487. 
35. Krack, M., Pseudopotentials for $\mathrm{H}$ to $\mathrm{Kr}$ optimized for gradient-corrected exchange-correlation functionals. Theor. Chem. Acc. 2005, 114 (1-3), 145-152.

36. Hahn, K. R.; Iannuzzi, M.; Seitsonen, A. P.; Hutter, J., Coverage effect of the CO2 adsorption mechanisms on CeO2(111) by first principles analysis. J. Phys. Chem. C 2013, 117 (4), 1701-1711.

37. Wang, Y. G.; Mei, D. H.; Li, J.; Rousseau, R., DFT+U study on the localized electronic states and their potential role during $\mathrm{H} 2 \mathrm{O}$ dissociation and $\mathrm{CO}$ oxidation processes on $\mathrm{CeO} 2(111)$ surface. $J$. Phys. Chem. C 2013, 117 (44), 23082-23089.

38. Tang, Y.; Zhao, S.; Long, B.; Liu, J. C.; Li, J., On the nature of support effects of metal dioxides $\mathrm{MO} 2(\mathrm{M}=\mathrm{Ti}, \mathrm{Zr}, \mathrm{Hf}, \mathrm{Ce}, \mathrm{Th})$ in single-atom gold catalysts: importance of quantum primogenic effect. J. Phys. Chem. C 2016, 120 (31), 17514-17526.

39. VandeVondele, J.; Hutter, J., Gaussian basis sets for accurate calculations on molecular systems in gas and condensed phases. J. Chem. Phys. 2007, 127 (11), 9.

40. VandeVondele, J.; Krack, M.; Mohamed, F.; Parrinello, M.; Chassaing, T.; Hutter, J., QUICKSTEP: Fast and accurate density functional calculations using a mixed Gaussian and plane waves approach. Computer Physics Communications 2005, 167 (2), 103-128.

41. Hutter, J.; Iannuzzi, M.; Schiffmann, F.; VandeVondele, J., CP2K: atomistic simulations of condensed matter systems. Wiley Interdisciplinary Reviews-Computational Molecular Science 2014, 4 (1), 15-25.

42. Hutter, J.; Ballone, P.; Bernasconi, M.; Focher, P.; Fois, E.; Goedecker, S.; Marx, D.; Parrinello, M.; Tuckerman, M. E., CPMD Version 3.9.1. Max Planck Institut Stuttgart and IBM Zurich Research Laboratory, 1990-2005.

43. Car, R.; Parrinello, M., Unified approach for molecular dynamics and density functional theory. Phys. Rev. Lett. 1985, 55 (22), 2471-2474.

44. ADF v2016.106, SCM http://www.scm.com: 2016.

45. Grimmel, S.; Schoendorff, G.; Wilson, A. K., Gauging the performance of density functionals for lanthanide-containing molecules. J. Chem. Theory Comput. 2016, 12 (3), 1259-1266.

46. Lu, Q.; Peterson, K. A., Correlation consistent basis sets for lanthanides: The atoms La-Lu. $J$. Chem. Phys. 2016, 145 (5), 13.

47. Frisch, M. J. T., G. W.; Schlegel, H. B.; Scuseria, G. E.; Robb, M. A.; Cheeseman, J. R.; Scalmani, G.; Barone, V.; Mennucci, B.; Petersson, G. A.; Nakatsuji, H.; Caricato, M.; Li, X.; Hratchian, H. P.; Izmaylov, A. F.; Bloino, J.; Zheng, G.; Sonnenberg, J. L.; Hada, M.; Ehara, M.; Toyota, K.; Fukuda, R.; Hasegawa, J.; Ishida, M.; Nakajima, T.; Honda, Y.; Kitao, O.; Nakai, H.; Vreven, T.; Montgomery, J. A., Jr.; Peralta, J. E.; Ogliaro, F.; Bearpark, M.; Heyd, J. J.; Brothers, E.; Kudin, K. N.; Staroverov, V. N.; Kobayashi, R.; Normand, J.; Raghavachari, K.; Rendell, A.; Burant, J. C.; Iyengar, S. S.; Tomasi, J.; Cossi, M.; Rega, N.; Millam, N. J.; Klene, M.; Knox, J. E.; Cross, J. B.; Bakken, V.; Adamo, C.; Jaramillo, J.; Gomperts, R.; Stratmann, R. E.; Yazyev, O.; Austin, A. J.; Cammi, R.; Pomelli, C.; Ochterski, J. W.; Martin, R. L.; Morokuma, K.; Zakrzewski, V. G.; Voth, G. A.; Salvador, P.; Dannenberg, J. J.; Dapprich, S.; Daniels, A. D.; Farkas, Ö.; Foresman, J. B.; Ortiz, J. V.; Cioslowski, J.; Fox, D. J., Gaussian 09. Gaussian, Inc., Walingford CT 2009.

48. Perdew, J. P.; Burke, K.; Ernzerhof, M., Generalized gradient approximation made simple. Phys. Rev. Lett. 1996, 77 (18), 3865-3868.

49. Woon, D. E.; Dunning, T. H., Gaussian basis stes for use in correlated molecular calculations. 3. The atoms aluminum through argon. J. Chem. Phys. 1993, 98 (2), 1358-1371.

50. van Lenthe, E.; Baerends, E. J.; Snijders, J. G., Relativistic total-energy using regular 
approximations. J. Chem. Phys. 1994, 101 (11), 9783-9792.

51. van Lenthe, E.; van Leeuwen, R.; Baerends, E. J.; Snijders, J. G., Relativistic regular twocomponent Hamiltonians. Int. J. Quantum Chem. 1996, 57 (3), 281-293.

52. van Lenthe, E.; Snijders, J. G.; Baerends, E. J., The zero-order regular approximation for relativistic effects: The effect of spin-orbit coupling in closed shell molecules. J. Chem. Phys. 1996, 105 (15), 6505-6516.

53. van Lenthe, E.; Ehlers, A.; Baerends, E. J., Geometry optimizations in the zero order regular approximation for relativistic effects. J. Chem. Phys. 1999, 110 (18), 8943-8953.

54. van Lenthe, E.; Baerends, E. J., Optimized slater-type basis sets for the elements 1-118. J. Comput. Chem. 2003, 24 (9), 1142-1156.

55. ADF STO basis set http://tc.chem.vu.nl/SCM/DOC/atomicdata/.

56. Goedecker, S.; Maschke, K., Transferability of pseudopotentials. Phys. Rev. A 1992, 45 (1), 88 93.

57. Dolg, M., Effective core potentials. Modern Methods and Algorithms of Quantum Chemistry 2000, 3, 507-540.

58. Desclaux, J. P., Citation classic - relativistic Dirac-Fock expectation values for atoms with $Z=1$ to $\mathrm{Z}=120$. Current Contents/Physical Chemical \& Earth Sciences 1981, (34), 16-16.

59. Gomes, A. P.; Dyall, K. G.; Visscher, L., Relativistic double-zeta, triple-zeta, and quadruple-zeta basis sets for the lanthanides La-Lu. Theor. Chem. Acc. 2010, 127 (4), 369-381.

60. Seitz, M.; Oliver, A. G.; Raymond, K. N., The lanthanide contraction revisited. J. Am. Chem. Soc. 2007, 129 (36), 11153-11160.

61. Mochizuki, Y.; Tatewaki, H., Four-component relativistic calculations on the complexes between a water molecule and trivalent lanthanoid and actinoid ions. Chem. Phys. 2001, 273 (2-3), 135-148. 62. Fomenko, V. S., Work function of yttrium and lanthanide single crystals. Powder Metall. Met. Ceram. 1994, 33 (1-2), 85-90.

63. Nemchenok, R. L.; Strakovskaya, S.; Titenskii, A., Energy distribution of photoelecytrons of gadolinium and terbium. Leningrad Polytechnical Institute, 1970.

64. Michaelson, H. B., Work function of elements and its periodicity. J. Appl. Phys. 1977, 48 (11), 4729-4733.

65. Lang, N. D.; Williams, A. R., Theory of local-work-function determinartion by photoemission from rare-gas adsorbates Phys. Rev. B 1982, 25 (4), 2940-2942.

66. Himpsel, F. J.; Reihl, B., Experimental energy bands of a rare-earth metal - Gd(0001). Phys. Rev. $B$ 1983, 28 (2), 574-578.

67. De Boer, F. R.; Mattens, W.; Boom, R.; Miedema, A.; Niessen, A., Cohesion in metals. NorthHolland: 1988.

68. Skriver, H. L.; Rosengaard, N. M., Surface energy and work function of elemental metals. Phys. Rev. B 1992, 46 (11), 7157-7168.

69. Kostikov, Y. I.; Dvoryankin, V., Work function of electron from low-index faces of bcc, fcc, and hcp metals with low indices. Zhurnal Fizicheskoj Khimii 1992, 66, 534-538.

70. Wyckoff, R. W., Hexagonal closest packed, hcp, structure. Cryst. Struct. 1963, 1, 7-83.

71. Kramida, A.; Ralchenk, Y.; Reader, J., NIST Atomic Spectra Database. National Institute of Standards and Technology: 2014. 


\section{TOC Graphic}

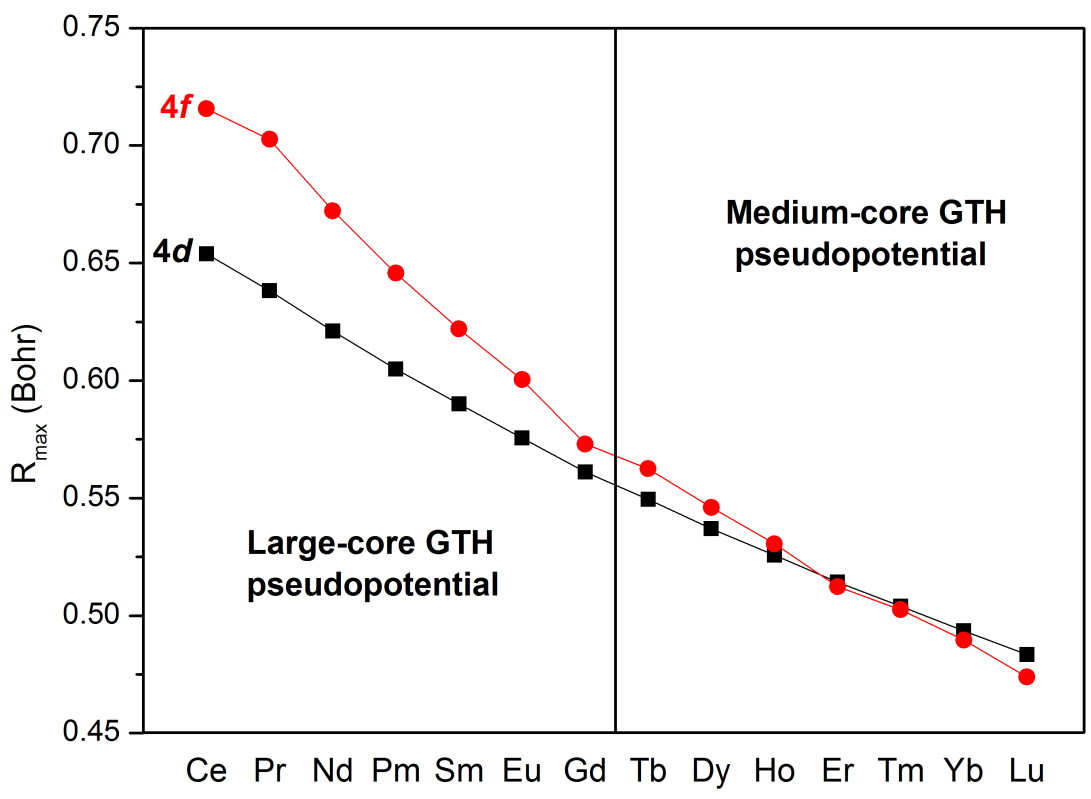

